\title{
Thermally activated dislocation mechanism in Mo studied by indentation, compression and impact testing
}

\author{
Christian Minnert ${ }^{1, a}{ }^{\oplus}$, Hamad ur Rehman ${ }^{1}$, Karsten Durst ${ }^{1}$ \\ ${ }^{1}$ Physical Metallurgy, Materials Science Department, Technical University of Darmstadt, Darmstadt, Germany \\ a) Address all correspondence to this author. e-mail: c.minnert@phm.tu-darmstadt.de \\ Received: 17 November 2020; accepted: 3 February 2021; published online: 23 February 2021
}

Body-centered cubic metals like molybdenum and tungsten are interesting structural materials for hightemperature applications. These metals, are however, brittle at low homologous temperature, caused by the limited mobility of screw dislocations. In this study, the thermally activated deformation mechanisms in bcc Mo have been investigated using strain rate jump nanoindentation and compression tests as well as Charpy V-notch impact testing. The material shows a significant softening with increasing temperature and a maximum in strain rate sensitivity is found at the critical temperature, before decreasing again in the ductile regime. The activation volume, however, showed a distinct increase from about $5 \mathbf{b}^{3}$ at the onset of the brittle to ductile transition temperature. Here we propose to use temperature-dependent nanoindentation strain rate jump testing and the activation volume as a complementary approach to provide some indication of the brittle to ductile transition temperature of bcc metals.

\section{Introduction}

Body-centered cubic (bcc), refractory metals like molybdenum or tungsten are interesting candidates for structural applications due to their high strength at elevated temperatures. The flow stress of these bcc metals at low homologous temperature is however mainly governed by the mobility of screw dislocations and therefore strongly dependent on temperature and applied strain rate. Thus, bcc metals exhibit a brittle behavior at low and a ductile behavior at elevated temperatures. The temperature at which the brittle to ductile transition (BDT) takes places is referred as the brittle to ductile transition temperature (BDTT). This well-studied behavior is caused by the crystal lattice and the activated slip systems:

In the low-temperature regime, the plastic deformation of bcc metals is mainly governed by the slip of $\frac{a}{2}\langle 111\rangle$ screw dislocations on $\{110\}$ planes, whereas at high temperatures, additional $\{112\}$ and $\{123\}$ slip planes are activated [1-4]. A spreading of the screw dislocation core on $\{110\}$ or $\{112\}$ planes can take place due to the threefold symmetry of the $\langle 111\rangle$ dislocation line [5]. In consequence, the Peierls potential or the Peierls-Nabarro barrier will increase, resulting in a higher resistance of motion of the screw dislocations. The strength of this barrier is reduced by thermal activation and the formation of kinks in the dislocation line. These kinks have an edge character and allow for the movement of dislocations over the barrier in steps. Thus, kink pairs are formed and they move through small kink steps instead of having to move the entire dislocation line at once. The nucleation and motion of these kink pairs is the rate controlling step, controlling the plastic deformation of bcc metals at low temperatures. Extrinsic effects like impurity atoms can constrain the kink mobility [3].

The dislocation velocity $v_{\mathrm{D}}$ is related to the applied plastic strain rate $\dot{\varepsilon}$ as described by the Orowan equation (Eq. 1) [5]:

$$
\dot{\varepsilon}=\rho_{\mathrm{m}} b v_{\mathrm{D}}
$$

with mobile dislocation density $\rho_{\mathrm{m}}$ and Burgers vector $b$. The screw dislocation velocity $v_{\mathrm{D}}$ is given by Eq. (2) [5]:

$$
v_{\mathrm{D}}=\rho_{\mathrm{K}} v_{\mathrm{K}} h,
$$

where $\rho_{\mathrm{K}}$ is the steady-state kink density, $v_{\mathrm{K}}$ the kink velocity and $\mathrm{h}$ the kink height. The kink pair nucleation rate $J$ as well as the kink velocity are thermal activated processes and can therefore be expressed by Arrhenius-type equations (Eqs. 3 and 4). The kink pair nucleation rate $J$ is [5]

$$
J=J_{0} \exp (-Q / \mathrm{kT})
$$


with a frequency constant $J_{0}$, required energy for kink pair nucleation $Q$, Boltzmann constant $k$ and absolute temperature $T$. It can be assumed that the energy required to initiate a kink movement $Q_{m}$ is significantly lower than the energy $Q$ required to form a kink pair. The resulting temperature-dependent kink velocity can be expressed by the following Arrhenius-type equation [5]:

$$
v_{\mathrm{k}}=2 a v \sinh (\tau \mathrm{bha} / \mathrm{kT}) \exp \left(-Q_{\mathrm{m} / \mathrm{kT}}\right)
$$

where parameter $\mathrm{a}$ is the distance which a kink is moving at each step, $v$ is a frequency constant and $\tau$ is the applied stress.

The plastic deformation is thus related to the formation and mobility of kinks. Both are thermally activated processes and therefore sensitive to test temperature and applied strain rate [6-9].

Seeger and Hollang [6] studied the temperature dependence of the flow stress of single-crystalline (SX) Mo. The data are shown in Fig. 1, with additional data from Cheng et al. [9], based on tensile tests carried out by Nemat-Nasser et al. [8] for coarse-grained (CG) Mo. It is apparent from the diagram that the flow stress of Mo consists of an athermal $\left(\sigma_{\mathrm{a}}\right)$ and a thermal component $\left(\sigma^{*}\right)$. The athermal component is related to the long-range internal stresses and microstructural barriers $[10,11]$. However, the aforementioned nucleation and motion of kink pairs of the screw dislocations affects the thermal component most and is responsible for the strain rate sensitivity (SRS) $\mathrm{m}$ of Mo. The flow stress of Mo decreases with increasing test temperature as shown in Fig. 1, until the knee temperature $T_{\mathrm{k}}$, also referred as athermal temperature, is reached and flow stress remains nearly constant $[7,9,12]$. The knee temperature is about $20 \%$ of the melting temperature for bcc metals [13]. For ultrapure single-crystalline Mo, a knee temperature of approx. $450 \mathrm{~K}$ was found [14]. Any further reduction of flow stress is

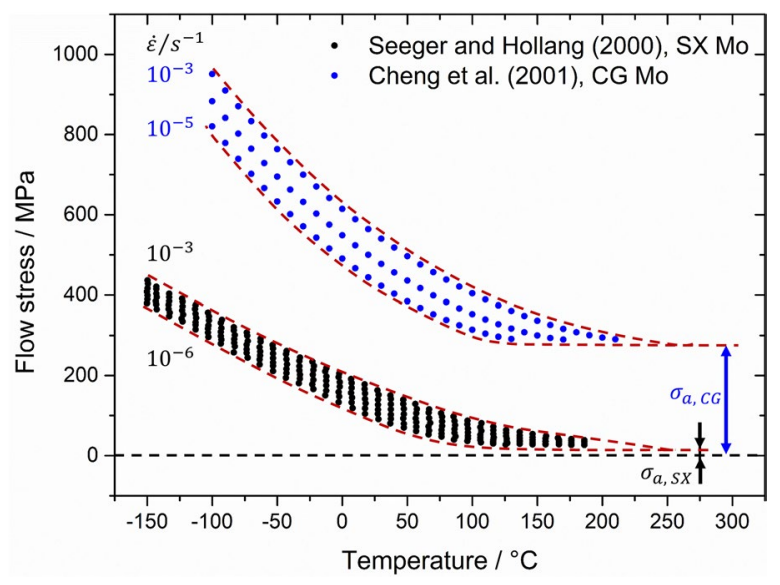

Figure 1: Temperature dependence of the flow stress of CG and SX Mo $[6,8,9]$. comparable to the temperature dependency of the shear modulus [9]. The deformation in the temperature range below $T_{\mathrm{k}}$ is controlled by the mobility of screw dislocations. It is apparent that the flow stress of the polycrystalline Mo from the tensile test data of Nemat-Nasser et al. $[8,9]$ is larger than that of singlecrystalline Mo which is related to an increase in the athermal stress component due to grain boundary strengthening (see Fig. 1). The data will be used later as reference and discussion of the temperature-dependent change in strain rate sensitivity and activation volume.

This strong increase in the thermal stress component of bcc metals and the enhanced rate sensitivity below $T_{\mathrm{k}}$ lead to brittle fracture at low temperatures. The BDT is controlled by thermally activated dislocation mobility [15-18] and thus related to the applied strain rate (Eq. 1), microstructure [12] and testing conditions [19]. In consequence, the BDTT is an extrinsic material parameter. It is currently not fully understood how the thermal activation and strain rate dependency of the flow stress governs the brittle to ductile transition temperature and how different testing methods could be used for estimating the temperature range for the BDT behavior of bcc metals.

In the present work, nanoindentation and compression testing are used to assess the correlation between thermally activated processes with respect to strain rate sensitivity and activation volume and the brittle to ductile transition temperature in bcc metals determined by conventional Charpy impact testing. All experiments were performed on polycrystalline Mo, which is used as reference material.

There are several publications dealing with the BDT or the temperature-dependent mechanical properties of Mo and its alloys [18, 20-27]. In the most studies, tensile or impact tests were carried out. However, the authors of this study are not aware of any work that has used such a wide range of investigative methods to characterize the brittle to ductile transition of bcc Mo, particularly in terms of the strain rate sensitivity and activation volume underlying dislocation mobility. Different strain rate and stress conditions have been achieved by employing diverse mechanical testing methods.

In Charpy impact tests, a high strain rate is present and a triaxial stress state is formed in the region of the notch [28]. In compression tests, on the other hand, a uniaxial stress state is reached and very low strain rates are applied [29]. Therefore, indentation strain rate jump tests were carried out for comparison, in which a triaxial stress state is generated within the plastic zone below the indenter tip [30], bearing some similarity to the impact tests.

The deformation mode observed during impact testing is controlled by dislocation emission from the crack tip, which in turn depends upon the stress state and testing temperature. This dislocation emission is a thermally activated process [6, 15], thus, the energy absorbed by the specimen during impact 
testing varies with temperature and strain rate. Furthermore, the dislocation mobility can lead to work hardening in the plastic zone next to the notch $[15,16,31]$. It is unclear how the thermally activated dislocation mobility changes in this high dislocation density region. In addition, different strengthening mechanisms, like grain boundaries or dislocation hardening, will influence the underlying mechanism and change the BDT behavior $[6,9,26,27]$.

The fracture properties, i.e., fracture toughness and BDTT of single-crystal bcc metals, vary with crystal orientation and active crack system. For tungsten, for instance, a change in BDTT of $100 \mathrm{~K}$ was found for different crystal orientations $[16,32]$. In polycrystalline materials, grain boundaries can represent a preferred crack path, where the fracture stress depends on the misorientation, respectively the grain boundary energy, of neighboring grains $[33,34]$. Segregations at the grain boundary can influence the grain boundary energy, resulting in an altered fracture behavior. Leitner et al. demonstrated how grain boundary segregations of carbon and boron change the fracture mechanisms from intergranular to transgranular resulting in a decreasing $\operatorname{BDTT}[35,36]$.

The active, temperature-dependent deformation mechanisms can be analyzed on the basis of the strain rate sensitivity (SRS) $\mathrm{m}$ and activation volume. Changing the strain rate within a single test opens the possibility to determine the strain rate sensitivity of a material in a quasi-constant volume element or microstructure. The relationship between applied strain rate $\dot{\varepsilon}$ and resulting flow stress $\sigma_{\mathrm{f}}$ or hardness $H$ is outlined in Eq. (5) [37]:

$$
m=\frac{d\left(\ln \sigma_{\mathrm{f}}\right)}{d(\ln \dot{\varepsilon})} \cong \frac{d(\ln H)}{d(\ln \dot{\varepsilon})}
$$

for uniaxial und indentation testing [37].

The activation volume $V$ is related to the process of kink formation when an obstacle is overcome by a jump of a dislocation line segment under an applied shear stress $\tau$ (Eq. 6) [38].

$$
V=\mathrm{kT}\left(\frac{\mathrm{d}\left(\ln \rho_{\mathrm{m}}\right)}{\mathrm{d} \tau}+\frac{\mathrm{d}\left(\ln v_{\mathrm{D}}\right)}{\mathrm{d} \tau}\right)
$$

The resulting activation volume can be calculated using Eq. (7) [37]:

$$
V=\frac{\sqrt{3} \mathrm{kT}}{m \sigma_{\mathrm{f}}} \cong 3 \sqrt{3} \frac{\mathrm{kT}}{m H}
$$

with Boltzmann constant $k$ and absolute temperature $T$ [37].

However, bcc metals show a well-known indentation size effect, i.e., the increase in hardness at low indentation depths, due to storage of geometrically necessary dislocations that are required to accommodate the large strain gradients induced by the indenter. This can lead to an overestimation of strain rate sensitivity [39, 40]. The Nix-Gao [41] analysis can be applied in order to get a depth-independent hardness value using Eq. (8):

$$
H=H_{0} \sqrt{1+\frac{h^{*}}{h}}
$$

with the hardness $H$ at an indentation depth $h$ and a characteristic length $h^{*}$. This characteristic length depends on the shape of the indenter, the sample shear modulus and the hardness level for an infinite depth $H_{0}$ [41]. However, the internal material length scale also changes with testing temperature [7].

This paper addresses the question whether quasi-static nanoindentation and compression tests can be used to study the temperature-dependent deformation mechanisms or in more particular the BDT and BDTT in bcc molybdenum as it is usually done with Charpy V-notch impact tests. Therefore, the strain rate sensitivities as well as the activation volumes were determined with different techniques causing different stress states in the material. The results are compared to literature data on single- and polycrystalline bcc Mo in terms of strain rate sensitivity and activation volumes.

\section{Results}

\section{Impact testing}

The absorbed energy determined in Charpy impact tests is plotted against temperature in Fig. 2a. A clear brittle fracture was observed at room temperature and below (Fig. 2b), with a low fracture energy in the order of $0.2 \mathrm{~J}$. This is typical of fracture devoid of plasticity at the crack tip [15]. A mixed mode fracture is observed in the intermediate temperature range between 75 ${ }^{\circ} \mathrm{C}$ and $110^{\circ} \mathrm{C}$. The cleavage crack propagated directly from the notch and followed the pancake microstructure (Fig. 2c). At $T>125^{\circ} \mathrm{C}$, the crack propagated in a ductile manner and plastic deformation was found next to the crack tip (Fig. 2d). A similar fracture behavior was observed by Babinsky et al. [27]. Dislocation slip becomes easier with an increase in temperature due to a reduction in the flow strength of Mo. Hence, more energy is absorbed by the samples in this regime. The brittle to ductile transition range is highlighted by the gray box in Fig. $2 \mathrm{a}$.

\section{Compression strain rate jump tests}

The results of compression strain rate jump tests in the brittle (RT), ductile $\left(200^{\circ} \mathrm{C}\right.$ ) and intermediate regimes (60 and $100{ }^{\circ} \mathrm{C}$ ) are shown in Fig. 3a. A change in strain rate leads to a short transient in stress, e.g., the first strain rate jump from $0.008 \mathrm{~s}^{-1}$ to $0.0008 \mathrm{~s}^{-1}$. This transition is caused by the mobility of the dislocation network as it is described by Eq. (1). Thermal activation of dislocations allows to overcome the Peierls barrier and the kink velocity increases (Eq. 4), resulting in a decrease in strength. The steady-state flow stresses at the constant strain 

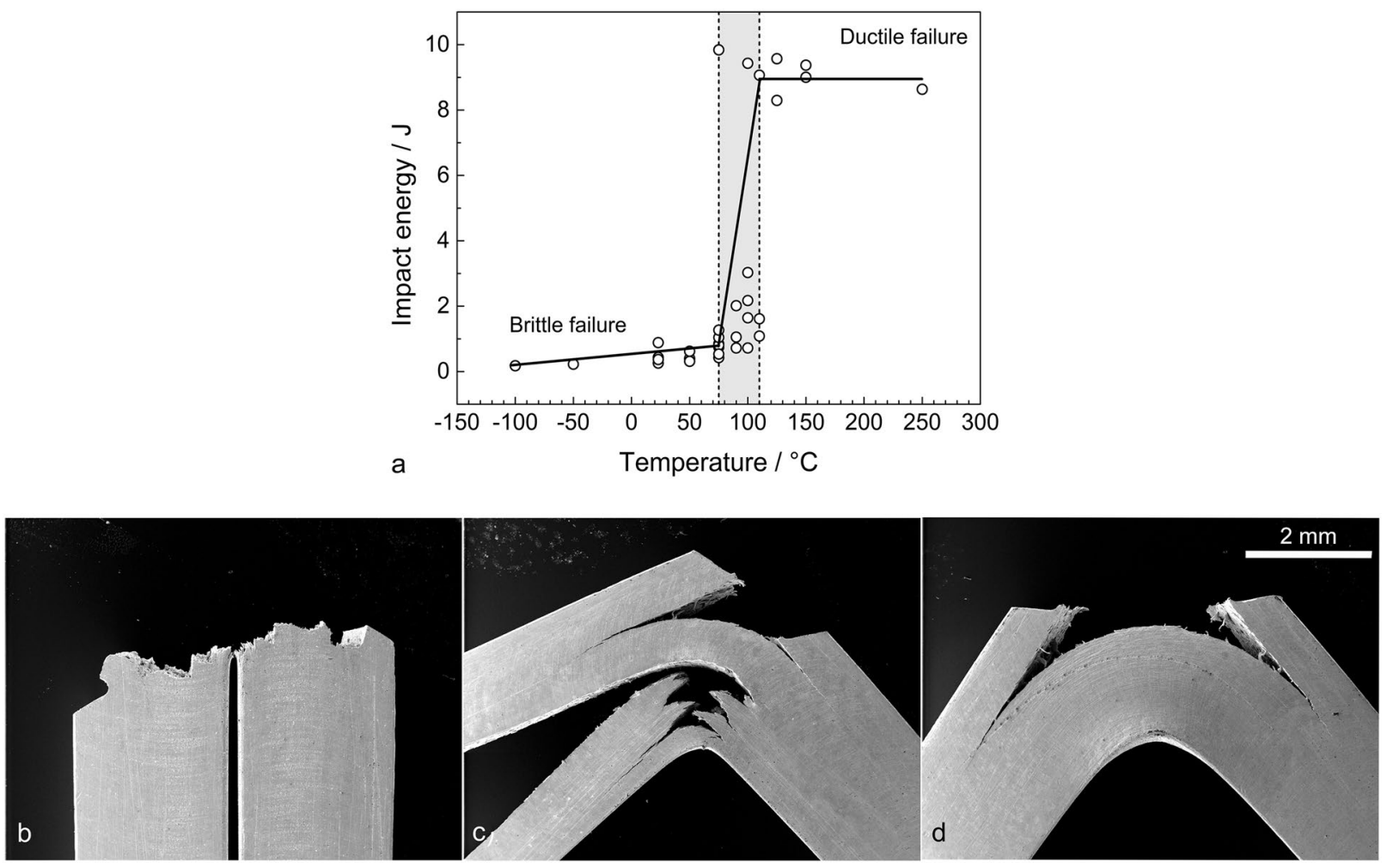

Figure 2: Charpy V-notch impact tests: (a) temperature-dependent energy absorbed by Mo samples during impact testing. The gray area indicates the brittle to ductile transition range $(b-d)$. Samples fractured tested at room temperature $(0.26 \mathrm{~J}), 100{ }^{\circ} \mathrm{C}(3.03 \mathrm{~J})$ and $250^{\circ} \mathrm{C}(8.64 \mathrm{~J})$.
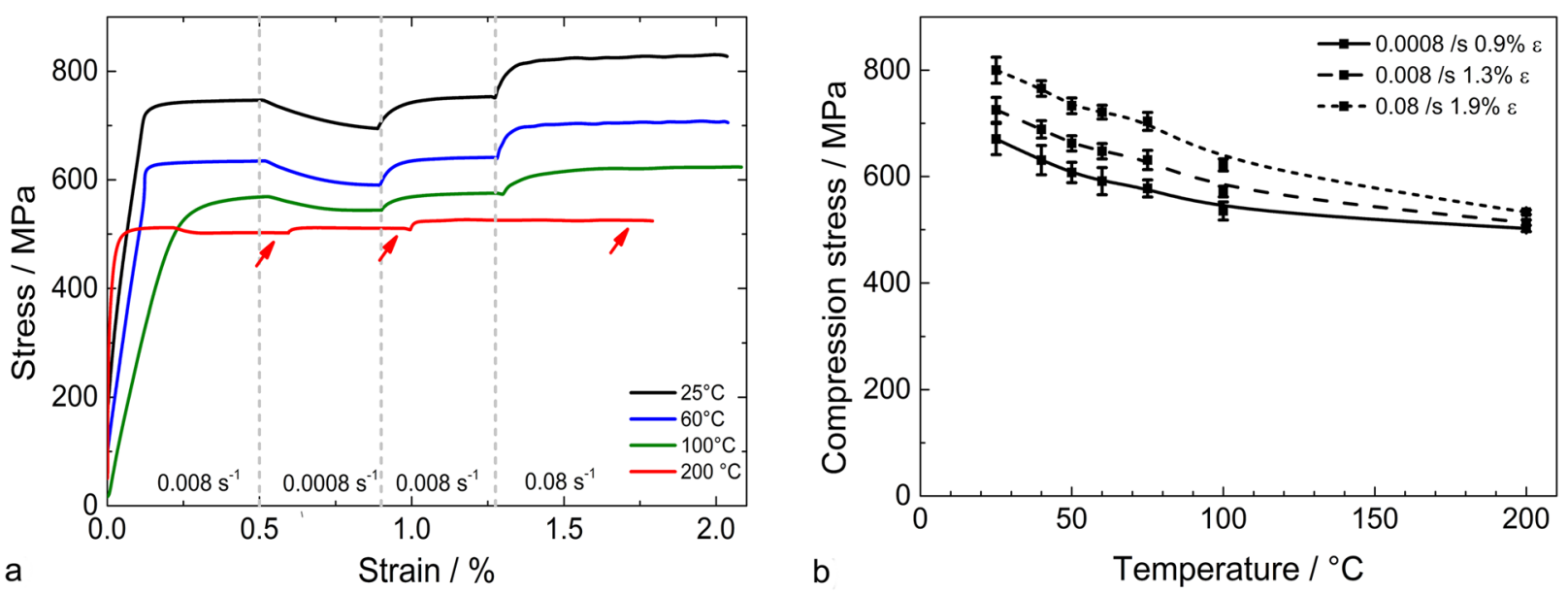

Figure 3: Compression strain rate jump tests: (a) stress over strain for different temperatures and (b) compression stress as a function of temperature for different strain rates.

rate marked by arrows (in Fig. 3a) are plotted as a function of temperature in Fig. 3b. These are later used to estimate the strain rate sensitivity and the activation volume.

\section{Indentation testing}

The load displacement curves of nanoindentation strain rate jump tests are shown in Fig. 4a. As mentioned above, the indentation size effect can lead to an overestimation of $\mathrm{m}$. Therefore, Nix-Gao [41] fitting of the hardness displacement plots was performed and the indentation size effect was subtracted from the depth-dependent hardness. This ensured that the estimated rate sensitivity and the activation volume are independent of the material length scale and indentation size effect. The resulting rate-dependent hardness is shown in Fig. 4b. During strain rate jump tests, a short transition is 

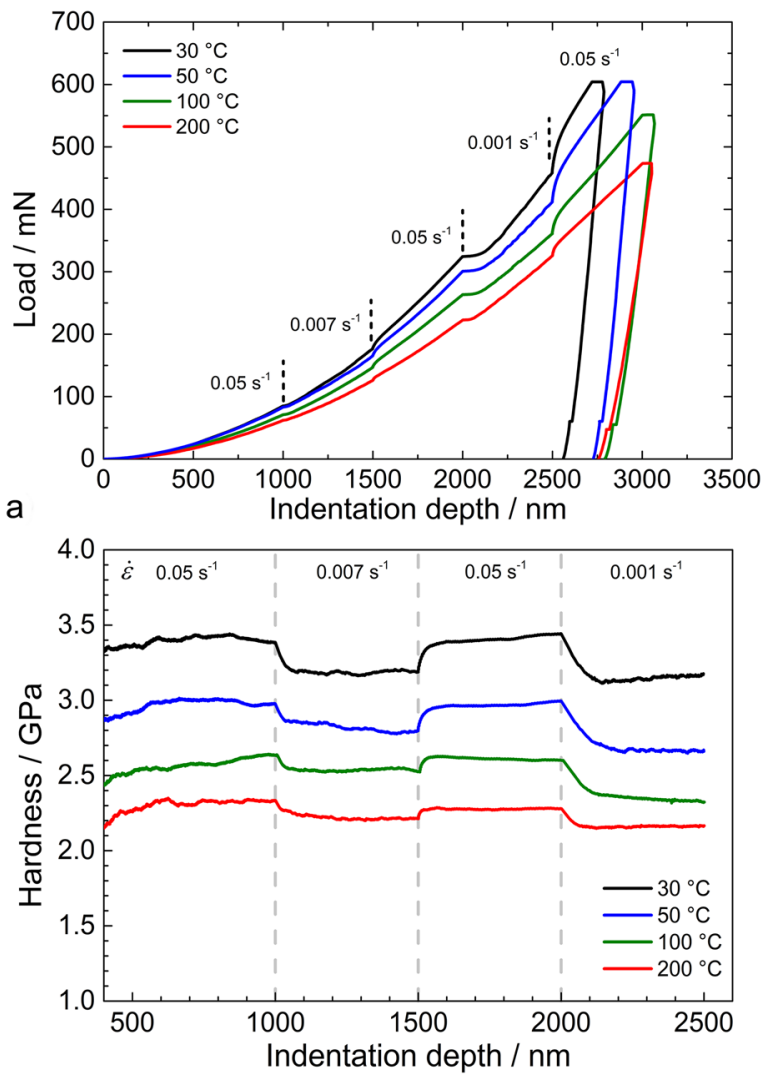

b

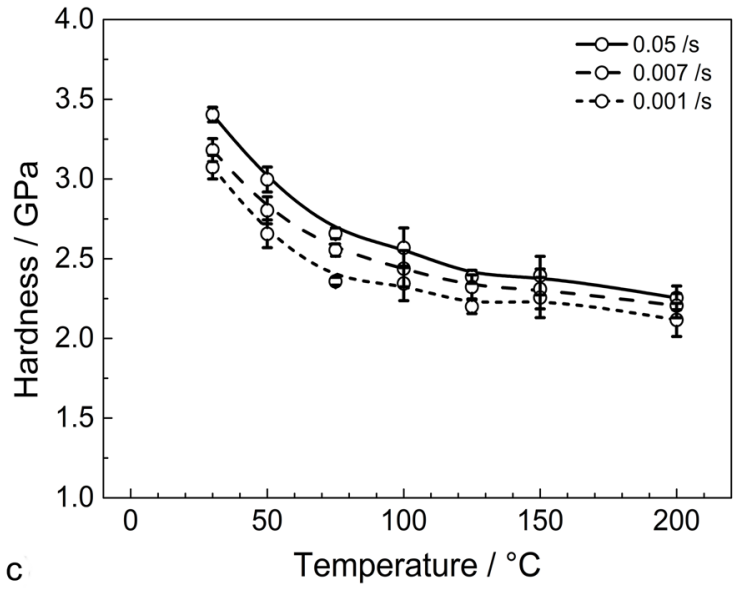

Figure 4: Nanoindentation tests: (a) load-displacement curve of the strain rate jump tests, (b) indentation size effect-corrected hardness from SRJ tests plotted against indentation depth, (c) hardness as a function of test temperature.

observed following a change in strain rate (Fig. 4b) similar to the compression SRJ tests.

The steady-state hardness from SRJ tests is decreasing with increasing test temperature as it is shown in Fig. 4c. It contains both the athermal and the thermal component of the flow stress, as the hardness can be converted into stress by multiplying with a constraint factor $\mathrm{C}$. The hardness does not decrease further with temperature beyond approx. $125^{\circ} \mathrm{C}$, indicating that the critical temperature has reached. The effect of deformation on the activation volume and the rate sensitivity will be discussed in the next section. Therefore, the strain rate sensitivity and the activation volume were estimated using Eqs. (5) and (7) [37, 38].

\section{Discussion}

Thermally activated processes in bcc Mo have been investigated by using low strain rate indentation and compression experiments at various temperatures. It is not fully understood how the high strain rates and stress triaxiality at the notch during an impact test will influence the underlying dislocation mechanisms. Such stress triaxiality is also found in nanoindentation testing and this work aims at establishing the possibilities offered by nanoindentation testing as an approach for investigating thermally activated processes at elevated temperatures. The results are compared with uniaxial compression tests and literature data. The triaxial stress state during indentation testing makes it difficult to directly compare hardness and compression flow stress values. Therefore, strain rate sensitivity coefficient $(m)$ is first used to compare results from different testing approaches.

The rate sensitivity coefficients and corresponding activation volumes-determined from present experiments, single-crystal data from Seeger and Hollang [6] and a modified mathematical model for polycrystalline Mo by Cheng et al. [9] - are plotted as a function of test temperature in Fig. 5. The brittle to ductile transition range determined by impact testing is highlighted in gray for comparison. The present measurements, as well as the literature data, show a small SRS at low temperatures and an increase in the rate sensitivity in the intermediate temperature range $\left(50-110^{\circ} \mathrm{C}\right)$ before again a decrease at higher temperatures ductile regime (Fig. $5 \mathrm{a}$ ). A similar behavior was also observed for bcc tungsten [42].

However, the absolute values of $\mathrm{m}$ are quite different for the different testing methods and microstructures. The highest SRS value is found for the single-crystal experiments by Seeger and Hollang [6], which is strongly increasing in the temperature range from approx. $50-110^{\circ} \mathrm{C}$. At this temperature range, the flow stress is strongly dropping with increasing temperature, however, the change in stress with strain rate remains largely constant.

The polycrystalline data by Cheng et al. [9] show a maximum in SRS in the temperature range from 50 to $80^{\circ} \mathrm{C}$. The SRSs determined from compression test data are almost identical to the literature values of Cheng et al., while the indentation technique provides significantly lower values. This indicates that the rate sensitivity is affected by the stress state inside the probed 

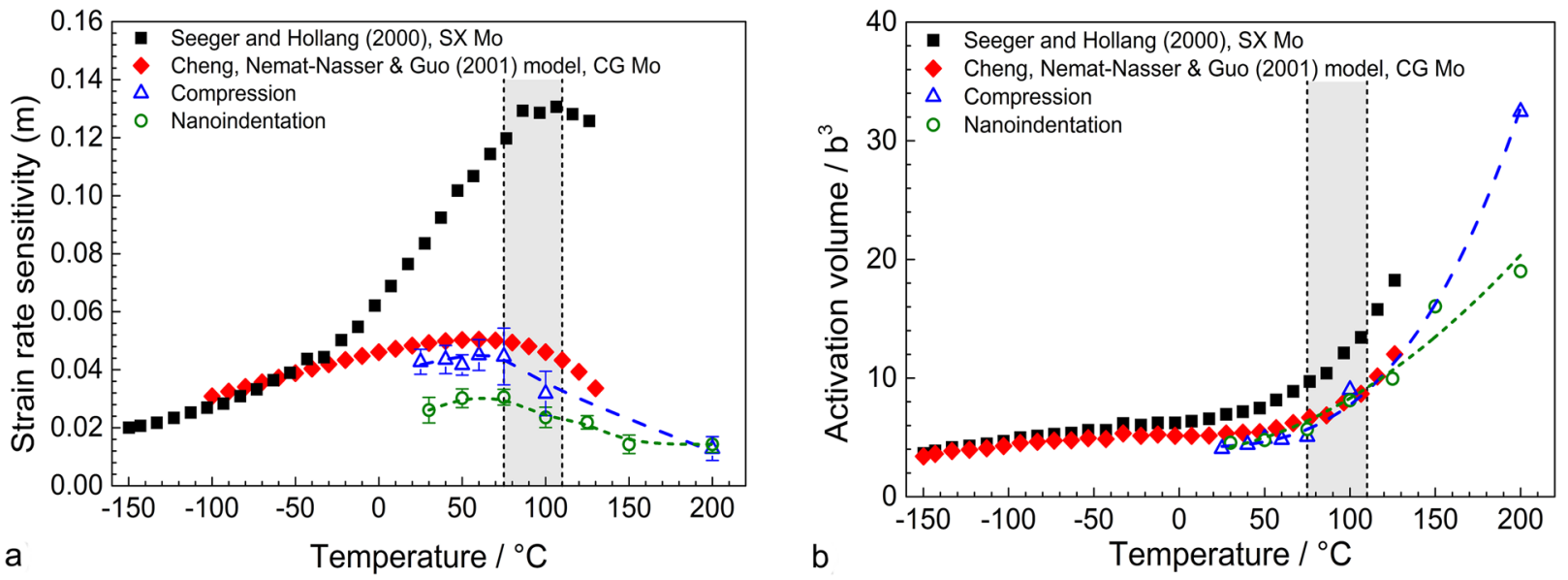

Figure 5: Temperature dependence of (a) strain rate sensitivity and (b) activation volume compared with literature from Seeger and Hollang [6] and mathematical model by Cheng et al. [8,9]. The brittle to ductile transition range from Charpy V-notch impact testing is highlighted in gray for comparison.

volumes, whereas the applied strain rate has a negligible impact on the results.

Such discrepancies between individual testing methods and applied strain rates are caused by the interplay between the net flow stress and the strain rate sensitivity. The flow stress consists of a thermal component (temperature dependent) and an athermal component (grain size dependent). Therefore, the flow stress of polycrystalline Mo is larger than that of singlecrystalline Mo due to grain boundary strengthening (see Fig. 1). Although the $\Delta \sigma / \Delta H$ is similar at a constant temperature, the lower overall value of flow stress values for the single-crystal specimen results in a larger value of the SRS coefficient when it is estimated using Eq. (5). Therefore, as the net flow stress of Mo increases by grain boundary strengthening, the strain rate sensitivity decreases.

The activation volumes determined for single- and polycrystalline Mo (Fig. 5b) however differ only slightly and the activation volume for polycrystalline Mo is almost independent of the test method (uniaxial or triaxial stress state during indentation). The activation volume gives thus a much better representation of the deformation mechanism under these test condition, since both rate sensitivity and flow stress respectively hardness are considered in the analysis (Eq. 7). In comparison, at low temperature an activation volume of about $5 \mathrm{~b}^{3}$ is found, and similar values $\left(4-7 \mathrm{~b}^{3}\right)$ are already reported for SX tungsten and chromium [43]. The activation volume is increasing to about 30 $\mathrm{b}^{3}$ at elevated temperatures.

Analyzing the temperature-dependent change of the activation volume in more detail, it is noticeable that a moderate, linear increase in the low temperature range can be observed. However, as soon as a critical temperature is reached, the gradient of the linear increase changes significantly. This behavior is shown in Fig. 6 in more detail for the different data sets. In the following analysis, the critical temperature is determined by a line intercept method and is thus called as intersection temperature, $T_{\text {int }}$, in the following. Therefore, the activation volumes for the low and high temperature range are fitted in their linear regime (red dashed lines) and the intersections of the linear fitting lines are used as a measure for $T_{\text {int }}$. This results in $T_{\text {int }}$ of approx. $77^{\circ} \mathrm{C}$ for the single-crystal data of Seeger and Hollang [4] and $88^{\circ} \mathrm{C}$ for the polycrystalline data of Nemat-Nasser [8] and Cheng et al. [9] (Fig. 6a, b). The same analysis for the SRJ tests yields an intersection temperature of $76^{\circ} \mathrm{C}$ for nanoindentation and $79^{\circ} \mathrm{C}$ for compression testing (Fig. 6c, d). Included in the diagram is also the range of the brittle to ductile transition temperature, as determined by the Charpy Impact test (Fig. 6c, d). It seems that the intersection temperatures are close to the lower limit of the BDT temperature range. At $T_{\text {int }}$, the SRS exhibits a maximum, whereas the activation volume is starting to increase significantly.

This leads to the question of the meaning of $T_{\text {int }}$ and the underlying physical processes, which are activated and lead to the strong change in the activation volume.

The intersection temperatures of $76-88{ }^{\circ} \mathrm{C}$ (Fig. 6) are significantly lower than the knee temperature of Mo single crystals, which is found to be $T_{\mathrm{k}}=177^{\circ} \mathrm{C}(450 \mathrm{~K})$ [14]. The knee temperature is not necessarily identical to the BDTT. It rather represents an upper limit for the BDTT, i.e., $T_{\mathrm{BDT}} \leq T_{\mathrm{k}}$ [44] as the Peierls barrier disappears at $T>T_{\mathrm{k}}$ and the material can deform in a ductile manner. At temperatures below $T_{\mathrm{k}}$, the deformation is limited by the mobility of the screw dislocations $[12,19]$. However, the mobility can already be high enough to cause a "semi-ductile" material behavior. The discussion above shows that the intersection temperature must be 

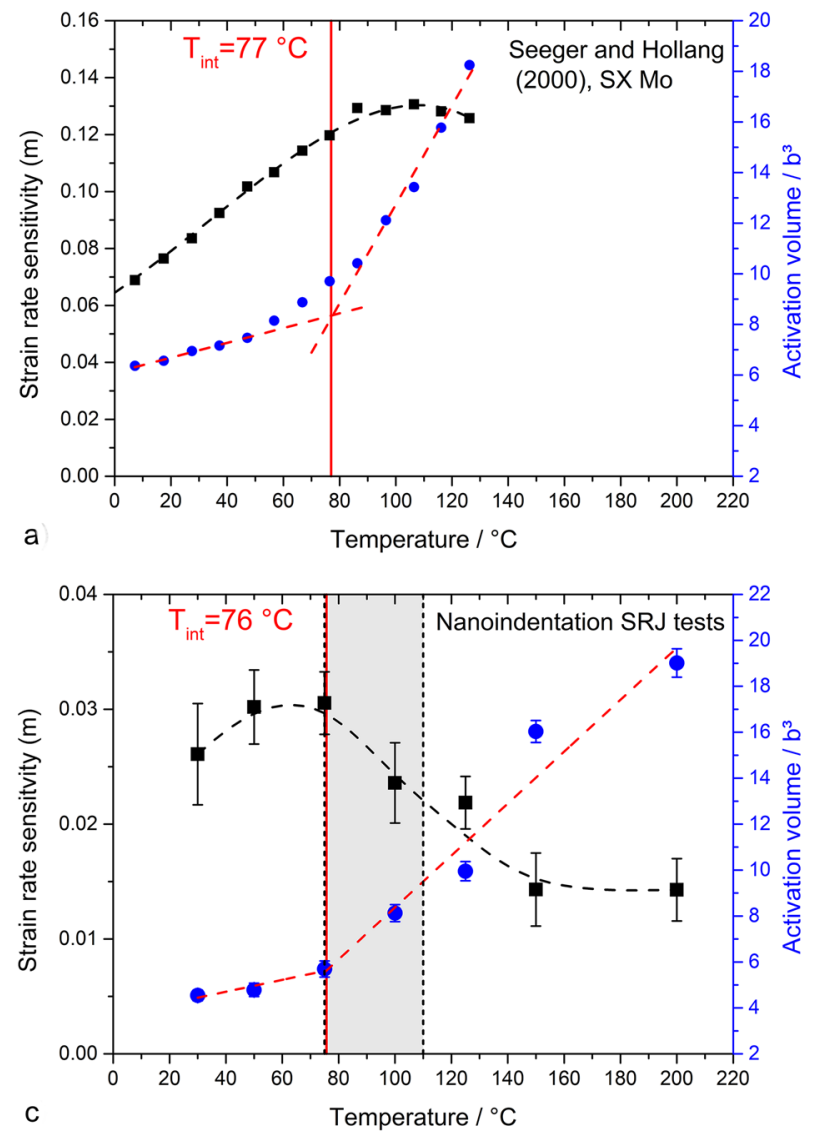
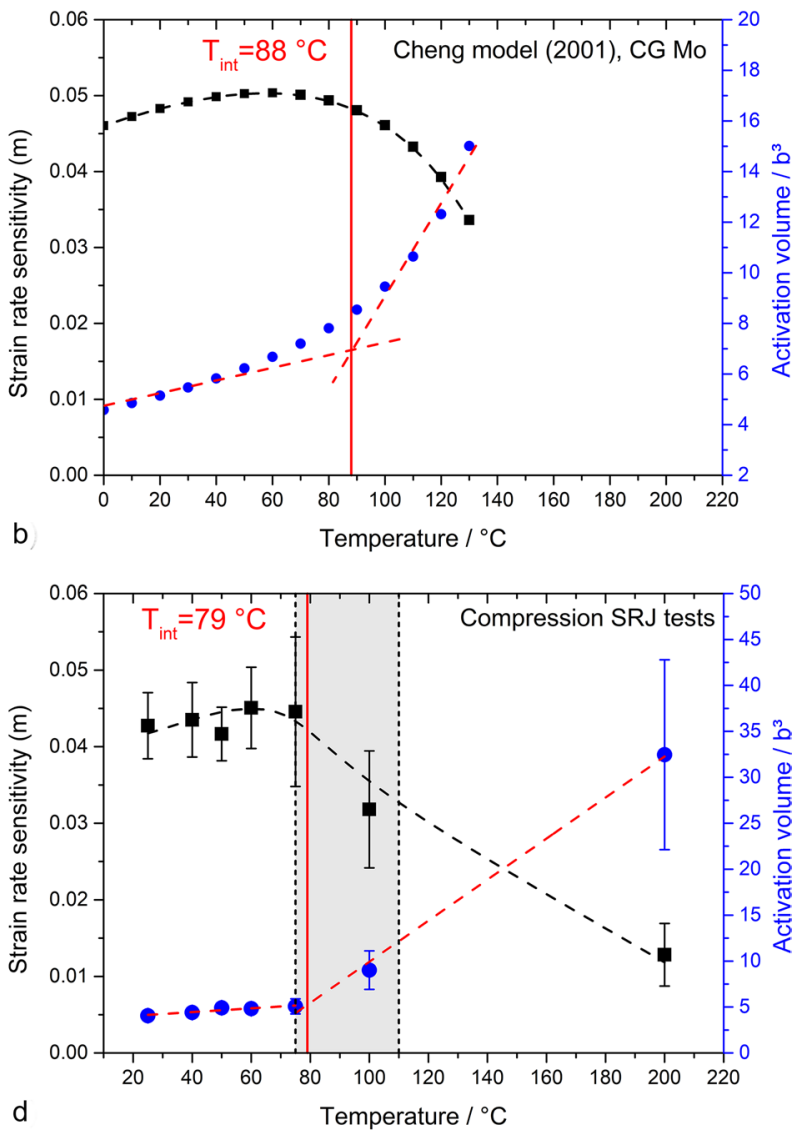

Figure 6: Determination of the intersection temperature for (a) SX Mo data from Seeger and Hollang [6], (b) Cheng-Nemat-Nasser model [8, 9], (c) nanoindentation strain rate jump tests and $\mathrm{d}$ ) compression strain rate jump tests. The brittle to ductile transition range from Charpy $\mathrm{V}$-notch impact testing is highlighted in gray for comparison.

below $T_{\mathrm{k}}$, so it has to be questioned how the BDTT is related to $T_{\mathrm{k}}$ and $T_{\mathrm{int}}$. At temperatures below $T_{\mathrm{int}}$, activation volumes of $V \leq 5 \mathrm{~b}^{3}$ are found, which can be linked to a kink mechanism [43]. At temperature above $T_{\mathrm{k}}$, the thermal energy alone is sufficient to overcome the Peierls barrier, the thermal component of the yield stress disappears (Fig. 1) and the mobility of screw and edge dislocations becomes equal $[12,19]$.

The $T_{\text {int }}$ is thus close to the onset of the BDTT and the very good agreement of literature data and the data provided here make us confident that by analyzing the intersection of the activation volumes an estimate of the BDTT can be provided.

It should be noted, however, that this rather simple analysis procedure can only give an approximation of the brittle to ductile transition temperature. The temperature-dependent course of $V$ can only be described with a linear fit to some extent. As shown in Fig. $6 c$, d, the data of this study show a well-defined change in $V$ with increasing $T$, while the literature data (Fig. 6a, b) show a rather continuous transition. This complicates the definition of appropriate fitting functions.
This discussion clearly shows that the SRS coefficient can only provide a qualitative description of the thermally activated processes. A much better understanding of the deformation mechanisms can be achieved by comparing the activation volumes, since in that case the SRS is normalized by the flow stress and therefore independent of the stress state. Furthermore, the activation volume can be directly related to the spacing between the obstacles, i.e., the smaller the distance between obstacles, the lesser will be the activation volume and vice versa $[45,46]$. Thus, the thermally activated mobility of screw dislocations is reflected in the activation volume. At low temperatures, the high Peierls stress does not allow the motion of dislocations and kinks must be nucleated for the dislocations to move resulting in a low activation volume of the order of $5 \mathrm{~b}^{3}$. At high temperatures, the deformation is controlled by the motion of kink pairs thereby resulting in a higher activation volume $\left(>20 \mathrm{~b}^{3}\right)$ as shown in Fig. 5b. This is in agreement with literature data [8]. For tungsten, an increase in $V$ to about $200 \mathrm{~b}^{3}$ was observed at 
$T>T_{\mathrm{k}}$, indicating dislocation interactions as observed in facecentered cubic materials $[42,43]$.

The most interesting finding of the present work is that irrespective of the testing method and stress state-triaxial in indentation and uniaxial compression testing - a similar change in the activation volume is observed with an increase in test temperature. This clearly shows that the fundamental deformation mechanism controlling plastic deformation is the same in all the testing methods and the activation volume is the controlling parameter, which must be analyzed to compare the different test methods. The applied strain rates during impact testing are significantly larger than those employed during conventional indentation testing. However, the stress triaxiality during impact testing and the plastic zone underneath the indenter tip are quite similar.

The different methods differ not only in the stress state and the applied strain rate, but also in the microstructure being tested. Seeger and Hollang have performed experiments on single-crystalline Mo and thus obtain best data for the movement of screw dislocations, while Nemat-Nasser et al. have investigated polycrystalline Mo. These results are influenced by the different crystal orientations and grain boundaries. The results of the impact and compression tests carried out in this work are also influenced by grain boundary effects due to the size of the plastic zones. This is not necessarily the case for nanoindentation testing, as the probed volumes are small in respect to the grain size. In consequence, the information is obtained from individual grains or from a small number of grains depending on the grain size of the tested material. Thus, the influence of boundary effects is not as pronounced as in the other testing methods carried out in this study.

It could be assumed that, in general, the indentation technique provides more reliable results than tensile or compression testing. This is especially the case for highly anisotropic materials, as there the hardness is less influenced by the grain structure and texture due to the triaxial stress state inside the plastic zone. Although the indentation data are less dependent on crystal orientation than uniaxial test results, the orientation can still affect $\mathrm{m}, \mathrm{H}$ and in consequence the activation volume as these parameters depend on the number of active slip systems [43]. Thus, the intersection temperature is expected to depend on the crystal orientation. Furthermore, it has been shown in the literature that Mo exhibits only a marginal orientation-dependent hardness [47].

For the present data, the influence of crystalline orientation has not been specifically addressed. However, due to the small scatter in data, a strong influence of the crystal orientation on the determination of the brittle to ductile transition is not expected.
Recently, the development of non-ambient temperature nanoindentation systems has made significant progress. This opens the possibility for studying the BDT in a rather wide temperature range between -142 and $1100^{\circ} \mathrm{C}[48,49]$.

In this study, the quasi-static tests show a slightly lower brittle to ductile transition temperature than Charpy impact tests with $93.5^{\circ} \mathrm{C}$ [middle of the transition range $\left.\left(75-110^{\circ} \mathrm{C}\right)\right]$. This is in accordance with literature where a strain rate dependence of the BDTT was observed $[15,16,26]$. This phenomenon is due to an increase of the yield strength with increasing strain rate [26]. Additionally, a local temperature increase can occur within the plastic zone, which is more pronounced for high deformation rates, since the adiabatic energy generated by plastic deformation cannot dissipate due to the short time scale. This effect can also cause a small shift in the transition temperature. Furthermore, the determination of an exact transition temperature by using impact testing is only possible to a limited extent due to the scattering of the data. It is therefore more favorable to specify a temperature range for the BDT. This shows a further advantage of the methodology presented here, where the increase in activation volume provides a clear indication of the BDT and thus a more precise, strain rate-dependent BDTT can be determined.

\section{Conclusions}

The active deformation mechanisms in bcc Mo have been investigated using different mechanical testing approaches and following conclusions could be drawn:

1. The absolute value of strain rate sensitivity parameter $(m)$ for bcc metals is dependent on the net flow stress. An increase in athermal component with grain boundary strengthening leads to a lower value of $m$ and vice versa.

2. Indentation and compression SRJ tests show a low strain rate sensitivity in the brittle regime. It increases with temperature to a maximum at the onset of the brittle to ductile transition before decreasing in the ductile regime.

3. The significant increase in the activation volume can be used in order to determine the brittle to ductile transition temperature and gives information about the active deformation mechanism. In addition, unlike yield stress/ hardness and strain rate sensitivity, it is independent of the microstructure and test method, since it depends only on the distance between thermal obstacles.

4. High-temperature nanoindentation strain rate jump tests can be used as a complementary approach to Charpy impact testing for investigating active deformation mechanisms 
and for estimation of the brittle to ductile transition temperature.

\section{Material and methods}

Polycrystalline Mo with a purity of $99.97 \%$ was purchased from Plansee $\mathrm{GmbH}$ as 5-mm-thick hot-rolled plates. The main impurities were tungsten $(<300 \mu \mathrm{g} / \mathrm{g})$, oxygen $(<40 \mu \mathrm{g} / \mathrm{g})$ and carbon with $(<30 \mu \mathrm{g} / \mathrm{g})$. These samples had a grain size of $125 \mu \mathrm{m}$ in the texture direction and a grain size of $37 \mu \mathrm{m}$ perpendicular to the texture direction.

Nanoindentation strain rate jump tests $[37,38]$ were performed using the load-controlled G200 nanoindenter (Keysight technologies, USA) equipped with a diamond Berkovich tip and a Laser heating set-up (both from Surface System Technology, Germany) for testing up to $500^{\circ} \mathrm{C}$. The sample and the indenter tip could be heated independently with a temperature stability of $0.1{ }^{\circ} \mathrm{C}$. A temperature adjustment procedure that ensures the accuracy of the tip and sample temperature is required for reliable measurements with low thermal drift rates at high temperatures [50]. The tip was therefore used as a probe by heating it with a constant laser power. Afterwards the sample temperature was adjusted until the change in tip temperature during contact with the sample was less than or equal to $0.1 \mathrm{~K}$. At least six tests were performed at 30, 50, 75, $100,125,150$ and $200{ }^{\circ} \mathrm{C}$. The test locations were chosen arbitrarily; nevertheless mainly the interior of different orientated grains was tested. Forming gas (VARIGON H5, Linde, Germany) was used to prevent oxidation of the tip and sample. Strain rate jump tests were used to investigate the thermally activated mechanisms by varying the applied strain rate in the range $0.05 \mathrm{~s}^{-1}$ to $0.001 \mathrm{~s}^{-1}$.

All indentation tests were performed on the same sample which was polished using the conventional metallographic polishing techniques followed by etching with a solution of $20 \mathrm{ml}$ $\mathrm{H}_{2} \mathrm{O}_{2}(30 \%)$ and $10 \mathrm{ml} \mathrm{H}_{2} \mathrm{SO}_{4}$ (95\%) in $70 \mathrm{ml}$ deionized water. Finally, the deformation layers-induced by mechanical polishing - were removed by vibrational polishing using an oxide particle-based solution.

Rectangular samples with dimensions of $4 \times 4 \times 8 \mathrm{~mm}^{3}$ were machined and polished plane-parallel for compression testing. Compression strain rate jump tests were performed using Z030 load frame (Zwick/Roell, Germany); it was equipped with a LVDT displacement sensor and a heating chamber, thus enabling measurements at $25,40,50,6075,100$ and $200{ }^{\circ} \mathrm{C}$. A preload of $250 \mathrm{~N}$ was applied to the samples before heating to the test temperature. The temperature was kept constant for $10 \mathrm{~min}$ before starting the test. This ensures a good thermal stability of the system. The strain rates during the jump tests consisted of $0.008 \mathrm{~s}^{-1}$ till a displacement of $45 \mu \mathrm{m}(0.5 \% \varepsilon), 0.0008 \mathrm{~s}^{-1}$ till $75 \mu \mathrm{m}(0.9 \% \varepsilon), 0.008 \mathrm{~s}^{-1}$ to $105 \mu \mathrm{m}(1.3 \% \varepsilon)$ and $0.08 \mathrm{~s}^{-1}$ till
$165 \mu \mathrm{m}(2 \% \varepsilon)$. Further details about the machine set-up and control of strain rate can be found in [51].

Miniaturized Charpy V-notch impact tests (KLST) were used to investigate the brittle to ductile transition at high strain rates. Impact tests were performed at Karlsruhe Institute of Technology (IAM-WBM) at different temperatures between -100 and $250^{\circ} \mathrm{C}$. The specimens were designed according to DIN 50,115. A notch with tip radius of $0.1 \mathrm{~mm}$ and opening angle of $60^{\circ}$ was machined in at the middle of the rectangular specimen with dimension of $27 \times 4 \times 3 \mathrm{~mm}^{3}$. Impact tests were carried out using an impact testing device (Zwick/Roell, Germany) with an impact energy of $25 \mathrm{~J}$ resulting in an impact velocity of $3.85 \mathrm{~m} / \mathrm{s}$. The striker radius is $2 \mathrm{~mm}$ and the spacing between the supports is $22 \mathrm{~mm}$. The system enables an automatic specimen cooling, heating and transporting in a test temperature range between -180 and $600^{\circ} \mathrm{C}$.

\section{Acknowledgments}

The authors gratefully acknowledge Dr. Ermile Gaganidze and Dr. Jarir Aktaa (both Karlsruhe Institute of Technology, Institute for Advanced Materials (IAM-WBM)) for the great support and for providing test equipment to perform the Charpy impact tests at elevated temperatures. Furthermore, Dr. Enrico Bruder, Dr. Sebastian Bruns, Dr. Kurt Johanns and Golo Zimmermann are thanked for experimental support. Deutsche Forschungsgemeinschaft (DFG) is gratefully acknowledged for financial support within the project No. 326946902. Additional financial support was provided by the Open Access Publication Fund of the Technical University of Darmstadt.

\section{Funding}

Open Access funding enabled and organized by Projekt DEAL.

\section{Data availability}

The data are available upon reasonable request from the corresponding author (C.M.).

\section{Compliance with ethical standards}

Conflict of interest The authors declare no conflict of interests.

\section{Open Access}

This article is licensed under a Creative Commons Attribution 4.0 International License, which permits use, sharing, adaptation, distribution and reproduction in any medium or format, as long as you give appropriate credit to the original author(s) and the source, provide a link to the Creative Commons licence, 
and indicate if changes were made. The images or other third party material in this article are included in the article's Creative Commons licence, unless indicated otherwise in a credit line to the material. If material is not included in the article's Creative Commons licence and your intended use is not permitted by statutory regulation or exceeds the permitted use, you will need to obtain permission directly from the copyright holder. To view a copy of this licence, visit http://creativecommons.org/ licenses/by/4.0/.

\section{References}

1. D. Hull, D.J. Bacon, Chapter 6-dislocations in other crystal structures, in Introduction to Dislocations, 5 th edn., ed. by $\mathrm{D}$. Hull, D.J. Bacon (Butterworth-Heinemann, Oxford, 2011), pp. $109-136$

2. V. Vitek, F. Kroupa, Dislocation theory of slip geometry and temperature dependence of flow stress in BCC metals. Phys. Status Solidi B 18(2), 703-713 (1966)

3. T. Suzuki, S. Takeuchi, H. Yoshinaga, Dislocations in bcc metals and their motion. in Dislocation Dynamics and Plasticity. (Springer, New York, 1991), pp. 77-98

4. V. Vitek, Core structure of screw dislocations in body-centred cubic metals: relation to symmetry and interatomic bonding. Philos. Mag. 84(3-5), 415-428 (2004)

5. M.S. Duesbery, G.Y. Richardson, The dislocation core in crystalline materials. Crit. Rev. Solid State Mater. Sci. 17(1), 1-46 (1991)

6. A. Seeger, L. Hollang, The flow-stress asymmetry of ultra-pure molybdenum single crystals. Mater. Trans., JIM 41(1), 141-151 (2000)

7. V. Maier et al., Microstructure-dependent deformation behaviour of bcc-metals-indentation size effect and strain rate sensitivity. Philos. Mag. 95(16-18), 1766-1779 (2015)

8. S. Nemat-Nasser, W. Guo, M. Liu, Experimentally-based micromechanical modeling of dynamic response of molybdenum. Scripta Mater. 40(7), 859-872 (1999)

9. J. Cheng, S. Nemat-Nasser, W. Guo, A unified constitutive model for strain-rate and temperature dependent behavior of molybdenum. Mech. Mater. 33(11), 603-616 (2001)

10. D.H. Lassila, A. Goldberg, R. Becker, The effect of grain boundaries on the athermal stress of tantalum and tantalum-tungsten alloys. Metall. Mater. Trans. A 33(11), 3457-3464 (2002)

11. G. Testa et al., Flow stress of bcc metals over a wide range of temperature and strain rates. Metals 10(1), 120 (2020)

12. C. Bonnekoh et al., The brittle-to-ductile transition in cold rolled tungsten plates: impact of crystallographic texture, grain size and dislocation density on the transition temperature. Int. J. Refract Met. Hard Mater. 78, 146-163 (2019)

13. A. Seeger, Why anomalous slip in body-centred cubic metals? Mater. Sci. Eng. A 319-321, 254-260 (2001)
14. L. Hollang, M. Hommel, A. Seeger, The flow stress of ultra-highpurity molybdenum single crystals. Phys. Status Solidi A 160(2), 329-354 (1997)

15. A. Hartmaier, P. Gumbsch, Thermal activation of crack-tip plasticity: the brittle or ductile response of a stationary crack loaded to failure. Phys. Rev. B 71(2), 024108 (2005)

16. P. Gumbsch et al., Controlling factors for the brittle-to-ductile transition in tungsten single crystals. Science 282(5392), 1293-1295 (1998)

17. A.A.N. Németh et al., The nature of the brittle-to-ductile transition of ultra fine grained tungsten (W) foil. Int. J. Refract Met. Hard Mater. 50, 9-15 (2015)

18. A.Y. Koval, A. Vasilev, S. Firstov, Fracture toughness of molybdenum sheet under brittle-ductile transition. Int. J. Refract Met. Hard Mater. 15(4), 223-226 (1997)

19. B.G. Butler et al., Mechanisms of deformation and ductility in tungsten-a review. Int. J. Refract Met. Hard Mater. 75, 248-261 (2018)

20. F. Fang, Y.Y. Zhou, W. Yang, In-situ SEM study of temperature dependent tensile behavior of wrought molybdenum. Int. J. Refract Met. Hard Mater. 41, 35-40 (2013)

21. A.S. Wronski, A.A. Johnson, The deformation and fracture properties of polycrystalline molybdenum. Philos. Mag. 7(74), 213-227 (1962)

22. A.S. Wronski, A.C. Chilton, E.M. Capron, The ductile-brittle transition in polycrystalline molybdenum. Acta Metall. 17(6), $751-755$ (1969)

23. L.P. Loshmanov, P.V. Fedotov, Effect of the strain rate on the mechanical properties of metalloceramic molybdenum in the ductile-brittle transition temperature range. Russ. Metall. 2009(5), 412-415 (2009)

24. J. Wadsworth et al., A microstructural investigation of the origin of brittle behavior in the transverse direction in Mo-based alloy bars. Metall. Trans. A 15(9), 1741-1752 (1984)

25. T. Takida et al., Mechanical properties of fine-grained, sintered molybdenum alloys with dispersed particles developed by mechanical alloying. Mater. Trans. 45(1), 143-148 (2004)

26. Y. Hiraoka et al., Fracture and ductile-to-brittle transition characteristics of molybdenum by impact and static bend tests. Mater. Trans., JIM 36(4), 504-510 (1995)

27. K. Babinsky et al., Fracture behavior and delamination toughening of molybdenum in Charpy impact tests. Jom 68(11), 2854-2863 (2016)

28. R.A. Wullaert, Applications of the Instrumented Charpy Impact Test Impact Testing of Metals (West Conshohocken, ASTM International, 1970), p. 164

29. Standard Test Methods of Compression Testing of Metallic Materials at Room Temperature.

30. A.C. Fischer-Cripps, Introduction to Contact Mechanics, vol. 101 (Springer, New York, 2007). 
31. E. Bitzek, P. Gumbsch, Mechanisms of dislocation multiplication at crack tips. Acta Mater. 61(4), 1394-1403 (2013)

32. P. Gumbsch, Brittle fracture and the brittle-to-ductile transition of tungsten. J. Nucl. Mater. 323(2-3), 304-312 (2003)

33. H. Kurishita et al., Misorientation dependence of grain boundary fracture in molybdenum bicrystals with various $\langle 110\rangle$ twist boundaries. Trans. Jpn. Inst. Met. 26(5), 332-340 (1985)

34. S. Tsurekawa, T. Tanaka, H. Yoshinaga, Grain boundary structure, energy and strength in molybdenum. Mater. Sci. Eng. A 176(1-2), 341-348 (1994)

35. K. Leitner et al., On grain boundary segregation in molybdenum materials. Mater. Des. 135, 204-212 (2017)

36. K. Leitner et al., Grain boundary segregation engineering in assintered molybdenum for improved ductility. Scripta Mater. 156, 60-63 (2018)

37. V. Maier et al., Nanoindentation strain-rate jump tests for determining the local strain-rate sensitivity in nanocrystalline $\mathrm{Ni}$ and ultrafine-grained Al. J. Mater. Res. 26(11), 1421-1430 (2011)

38. K. Durst, V. Maier, Dynamic nanoindentation testing for studying thermally activated processes from single to nanocrystalline metals. Curr. Opin. Solid State Mater. Sci. 19(6), 340-353 (2015)

39. G.M. Pharr, E.G. Herbert, Y. Gao, The indentation size effect: a critical examination of experimental observations and mechanistic interpretations. Annu. Rev. Mater. Res. 40, 271-292 (2010)

40. K. Durst et al., Indentation size effect in metallic materials: modeling strength from pop-in to macroscopic hardness using geometrically necessary dislocations. Acta Mater. 54(9), 2547-2555 (2006)

41. W.D. Nix, H. Gao, Indentation size effects in crystalline materials: a law for strain gradient plasticity. J. Mech. Phys. Solids 46(3), 411-425 (1998)
42. D. Brunner, V. Glebovsky, Analysis of flow-stress measurements of high-purity tungsten single crystals. Mater. Lett. 44(3-4), 144-152 (2000)

43. D. Kiener et al., Rate limiting deformation mechanisms of bcc metals in confined volumes. Acta Mater. 166, 687-701 (2019)

44. V. Trefilov, Y.V. Milman, I. Gridneva, Characteristic temperature of deformation of crystalline materials. Cryst. Res. Technol. 19(3), 413-421 (1984)

45. U. Kocks et al., Prog. Mater. Sci. 19, 1-281 (1975)

46. D. Caillard, J.L. Martin, Thermally Activated Mechanisms in Crystal Plasticity. Pergamon Materials Series, ed. R.W. Cahn. vol. 8 (2003)

47. S. Jakob et al., Influence of crystal orientation and Berkovich tip rotation on the mechanical characterization of grain boundaries in molybdenum. Mater. Des. 182, 107998 (2019)

48. A. Barnoush et al., In situ small-scale mechanical testing under extreme environments. MRS Bull. 44(6), 471-477 (2019)

49. C. Minnert, W.C. Oliver, K. Durst, New ultra-high temperature nanoindentation system for operating at up to $1100^{\circ} \mathrm{C}$. Mater. Des. 192, 108727 (2020)

50. J.M. Wheeler et al., High temperature nanoindentation: the state of the art and future challenges. Curr. Opin. Solid State Mater. Sci. 19(6), 354-366 (2015)

51. E.A. Patterson et al., Temperature-dependent deformation and dislocation density in $\mathrm{SrTiO}_{3}$ (001) single crystals. J. Am. Ceram. Soc. 99(10), 3411-3420 (2016) 